\title{
Evaluation of Water Quality and Benthic Macrointervebrates Fauna Relationship Using Principal Component Analysis (PCA): A Case Study of Cameron Highlands Malaysia
}

\author{
Kok Weng Tan (Corresponding author) \\ Department of Environmental Engineering, Faculty of Engineering and Green Technology \\ Universiti Tunku Abdul Rahman, 31900 Kampar Perak, Malaysia \\ Email: tankokweng@utar.edu.my
}

Weng Chee Beh

Faculty of Science and Technology, Universiti Kebangsaan Malaysia

43600 Bangi Selangor, Malaysia

Received: February 16, 2016 Accepted: March 9, 2016

doi:10.5296/emsd.v5i1.9399 URL: http://dx.doi.org/10.5296/emsd.v5i1.9399

\begin{abstract}
This study applies the Principal Component Analysis (PCA) to evaluate and interpret the relationship between water quality and benthic macro-invertebrates fauna data obtained from Pauh River, Cameron Highlands. Samples were collected once every two months (in February, April, June, August and October 2013) with six chosen sampling stations. Six water quality parameters namely dissolved oxygen (DO), $\mathrm{pH}$, biological oxygen demand (BOD 5 ), chemical oxygen demand (COD), ammonia-nitrogen $\left(\mathrm{NH}_{3}-\mathrm{N}\right)$, total suspended solid (TSS) and heavy metals contents were analyzed according to American Public Health Association (APHA), Standard Methods for Examination of Water and Wastewater (1998). Macro-invertebrates were also sampled using Surber sampler and were identified until their family level. Water Quality Index (WQI) values for all stations were class II except for the station 6 which was recorded as class III. Both the diversity and biotic indices showed decreasing value from the upstream (Station 1) to downstream (Station 6). A total 28 to 31 taxa have been found in Station 1, 2, 3 and 5 (upstream to middle stream). However, only 7 taxa found at station 6 (downstream). Total 31 taxa with an average density $368.28 \mathrm{ind} / \mathrm{m}^{2}$ were found in Station 4
\end{abstract}


which was highest number of taxa among the monitoring stations. The principal component analysis (PCA) was applied on the dataset, which explained $72.15 \%$ of the total variance of the variables. Three components were extracted in this study. First component was classified as benthic macroinvertebrates which tolerated to low water quality condition and high loading of organic matters. The benthic macro-invertebrates families loaded in second component were sensitive to water environment such as $\mathrm{NH}_{3}-\mathrm{N}$, dissolved oxygen (DO), organic matter and stream flow. The benthic macroinvertebrate families loaded in third component were recognized as species which might not tolerate low concentration of dissolved oxygen.

Keywords: Water quality; benthic macro-invertebrates, bio-indicator, principal component analysis, Cameron Highlands, Malaysia

\section{Introduction}

Human activities have severely affected the condition of freshwater ecosystems worldwide. Physical alteration, habitat loss, water withdrawal, pollution, overexploitation and the introduction of non-native species all contribute to the decline in freshwater species and the water quality as well. Increasing human population growth and achieving higher development targets place even higher demand on the already stressed freshwater ecosystems. Water quality is a measurement to determine the pollution level that happens in water, showing the reaction in water composition towards all the input whether is natural or manmade (Karanth 1987; Krenkel \& Novotny 1980). However, physical and chemical monitoring instruments are usually expensive and can only be used at limited number of sites thus unable to achieve distribution patterns (Swaminathan 2003). Hence, Biological monitoring is considered one of the alternatives which is a useful and rapid assessment tool to check the status of water quality.

Biological monitoring (also called bio-monitoring or bio-assessment) is defined as an evaluation of the condition of a water body using biological surveys and other direct measurements of the resident biota in surface waters (Engel and Voshell 2002) for example plants and animals or its components to provide continuous analytical information (Kopciuh et al. 2004). Biological monitoring can be done with any living organisms (biological indicators) but benthic macroinvetebrate, fish, and periphyton (algal) assemblages are used more often, in that order (Engel and Voshell 2002). Those biological indicators describing the condition and threats to freshwater ecosystems are required to measure progress in halting the rapid decline in freshwater species (Revenga et al. 2005). Tolerance of bio-indicator organism usually have its limit, therefore the presence or absence and its health state can determine some of the chemical and physical components in the environment without the complex measurement and laboratory work (Kopciuh et al. 2004). Changes in benthic macroinvertebrates community with water pollution have many been documented and measured using different aspects including biomass, species density and species composition (Yong et al. 1997).

Macroinvertebrates are found in even the smallest streams and have a wide range of sensitivity to all types of pollution and stress, allowing for monitoring in most conditions 
(Tan \& Beh 2015). Apparently, sampling of macroinvertebrates is easy, cost effective, and does not permanently harm the local assemblage (Engel and Voshell 2002; Kusza, 2005). Impairment can be easily be detected by the trained monitor with even the simplest of identifications (Engel and Voshell 2002). Hamed et al. (2014) states that more species distribution indicated (i.e. the number of individual organisms or abundance of species are more similar the level of stability of biodiversity in the area. This distribution of species can be determined by different types of bio-indices e.g. Shannon Diversity Index, Margalef Diversity index and etc. Different types of studies have been conducted on the relationship of water quality and bio-indices i.e. Hamed et al. (2014), Azrina et al. (2006), Ogleni and Topal (2011), Nemati et al. (2010), Latha and Thang (2010). However, field survey and laboratory analysis usually generates a large data set. This large and complex survey data is not easy to be interpreted and understood by environmental manager. In order to overcome this difficulty, multivariate methods are applied to develop an interpretability model for decision maker. Factor analysis (FA) such as principal component analysis (PCA) and principal factor analysis (PFA) have been recognized as powerful methods that could be used to reduce the dimensionality of a data set consisting of large number of inter-related variables, while retaining as much as possible the variability present in dataset (Einax et al. 1998). This reduction is achieved by transforming the data set into a new set of variable (Singh et al. 2004). Identification of the significant parameters and characteristic change by data reduction and interpretation in variables can be approached through the application of factor analysis. Thus, the aim of this study is to determine the relationship between river water quality and the distribution of benthic macroinvertebrates organism in the Cameron Highlands.

\section{Study Area}

Cameron Highlands is a well-known tourism destination and agricultural place of Malaysia, but also an environmentally-sensitive area (Wan Abdullah et al. 2005; Gasim et al. 2009). It is situated in the Pahang State and has an estimated area of $712 \mathrm{~km}^{2}$. It is located on the Main Range of Peninsular Malaysia between $4^{\circ} 20^{\prime} \mathrm{N}-4^{\circ} 37^{\prime} \mathrm{N}$ and $101^{\circ} 20^{\prime}-101^{\circ} 36^{\prime} \mathrm{E}$ (Figure 1), which is made up of three major sub-district of Telom, Ringlet and Tanah Rata. The altitude is between 1,280 $\mathrm{m}$ and 1,830 $\mathrm{m}$ above sea level (Ngan et al. 2005; Gasim et al. 2009). Due to highland characteristics, Cameron Highlands has average daily temperature is about $14{ }^{\circ} \mathrm{C}$ to $21^{\circ} \mathrm{C}$ and annual rainfall is $2,800 \mathrm{~mm}$ (Ismail et al. 2004; Gasim et al. 2009). With these unique characteristics, agricultural cultivation becomes the main human activity in Cameron Highlands. However, unsustainable agricultural practices also have been recognised as main pollution sources to water quality in this area (Tan 2010).

Three main rivers have been identified in Cameron Highlands namely Bertam River, Telom River and Lemoi River. An estimated 5.8 million litres per day (MLD) of water is abstracted at several water supply intake points from rivers originating from mountain forests in Cameron Highlands for water supply and irrigation purpose (Kumaran and Ainuddin 2006) and Pauh River is one of tributaries for Bertam River. According to Dinakaran \& Anbalagan (2007), Pauh River was identified as one of important recreational place for both local and international tourist. 


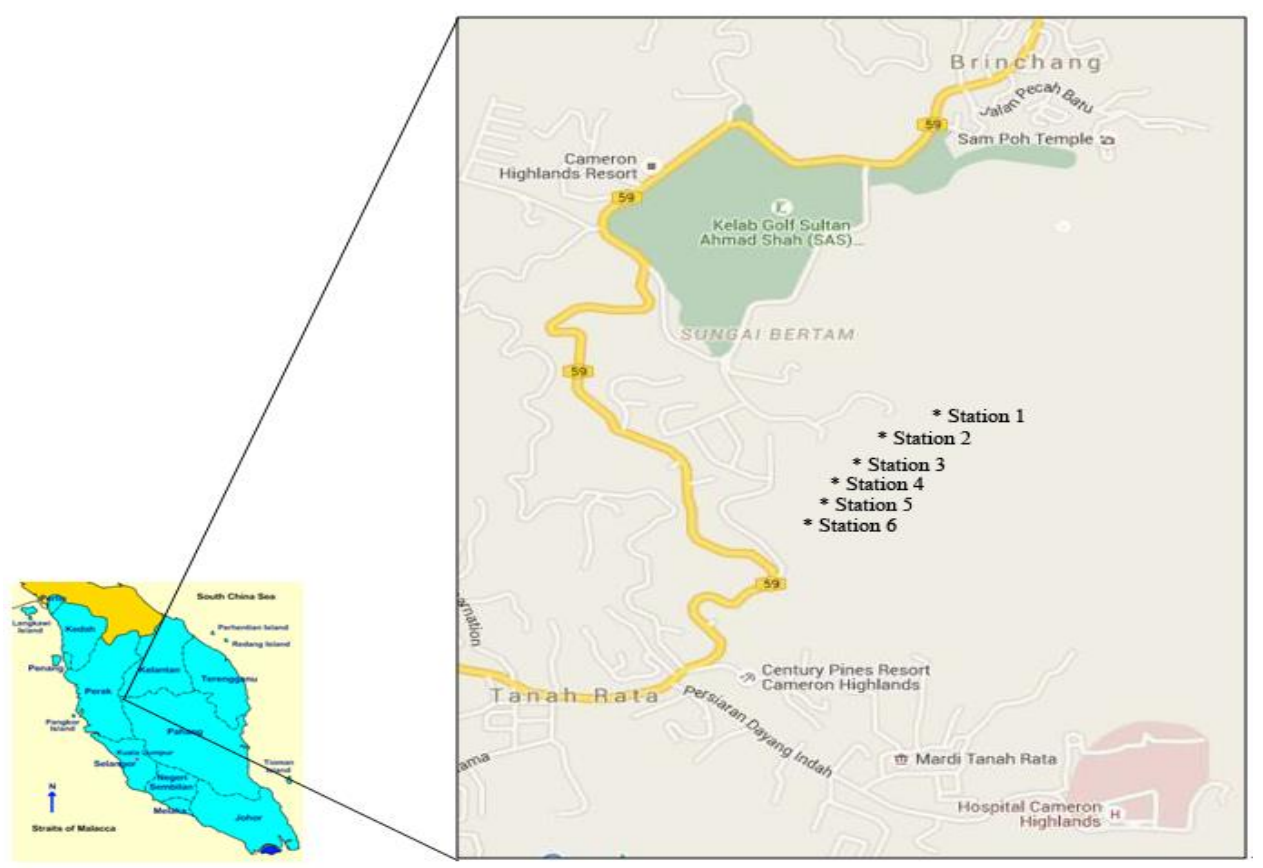

Figure 1. Location of Monitoring Station in Cameron Highlands in Malaysia

(Source: JUPEM 2012)

\section{Experimental Analysis}

Total six sampling stations were set up for this study which representing upstream and downstream of Pauh River. Characteristics of monitoring stations were shown in Table 1. Water quality and macroinvertebrates organism samplings were conducted once every 2 months throughout the year of 2015. The geographical records of the sampling site were taken using Global Positioning System (GPS). Hydrological and ecological information such as river bed composition, width, depth, stream flow and general description of the sampling site was noted by field investigation.

Table 1. Characteristics of monitoring stations in Pauh River, Cameron Highlands

\begin{tabular}{|c|c|c|c|c|c|c|}
\hline Sompling ctation & 1 & 2 & 3 & 4 & 5 & 6 \\
\hline Location & $\begin{array}{l}\mathrm{N} \quad 04^{\circ} 28^{\prime} \\
47^{\prime \prime} \\
\text { E } 101^{\circ} 23^{\prime} \\
6.4^{\prime \prime}\end{array}$ & $\begin{array}{l}\mathrm{N} \quad 04^{\circ} \quad 28^{\prime} \\
48.4^{\prime \prime} \\
\text { E } 101^{\circ} \quad 23^{\prime} \\
7.5^{\prime \prime}\end{array}$ & $\begin{array}{l}\mathrm{N} 04^{\circ} 28^{\prime} \\
6.3^{\prime} \\
\text { E } 101^{\circ} 23^{\prime} \\
7.8^{\prime \prime}\end{array}$ & $\begin{array}{l}\mathrm{N} \quad 04^{\circ} \quad 28^{\prime} \\
6.3^{\prime \prime} \\
\mathrm{E} \quad 101^{\circ} 22^{\prime} \\
59.1^{\prime \prime}\end{array}$ & $\begin{array}{l}\mathrm{N} \quad 04^{\circ} \quad 28^{\prime} \\
45^{\prime \prime} \\
\mathrm{E} \quad 101^{\circ} 22^{\prime} \\
57.9^{\prime \prime}\end{array}$ & $\begin{array}{lll}\mathrm{N} & 04^{\circ} & 28^{\prime} \\
45^{\prime \prime} & \\
\mathrm{E} & 101^{\circ} & 22^{\prime} \\
57.06^{\prime \prime} & \end{array}$ \\
\hline Elevation (m) & 1477 & 1449 & 1501 & 1463 & 1451 & 1468 \\
\hline River length (m) & 3.3 & 3.4 & 1.5 & 1.7 & 2.0 & 6.7 \\
\hline River depth (m) & 0.16 & 0.18 & 0.14 & 0.16 & 0.15 & 0.32 \\
\hline Stream flow $\left(\mathrm{ms}^{-1}\right)$ & $1.0 \pm 0.5$ & $1.0 \pm 0.5$ & $0.8 \pm 0.3$ & $0.8 \pm 0.3$ & $0.5 \pm 0.1$ & $0.3 \pm 0.1$ \\
\hline Vegetation (\%) & 90 & 80 & 60 & 20 & 30 & 10 \\
\hline
\end{tabular}




\begin{tabular}{|l|c|c|c|c|c|c|}
\hline River subtract & & & & & \\
compositions & & & & & & \\
i. Rock & 80 & 60 & & & & \\
ii. Sand & 15 & 30 & 50 & 50 & 40 & 20 \\
iii. Mud & 5 & 10 & 10 & 20 & 40 & 30 \\
\hline
\end{tabular}

\subsection{Water Sampling and Macroinvertebrates Analysis}

Measurement of temperature, $\mathrm{pH}$, dissolved oxygen (DO), conductivity and total dissolved solid was conducted during field investigation by using multi-parameters YSI 556 MPS (APHA 1992; APHA 1998). All these parameters were measured $1 \mathrm{~m}$ from the water surface because at this depth, the content of the stream water are mixing well. Water sample for detection of biological oxygen demand (BOD), chemical oxygen demand (COD), ammoniacal-nitrogen $\left(\mathrm{NH}_{3}-\mathrm{N}\right)$ and total suspended solid (TSS) were taken and analyzed by referring the standard method of APHA 1998. Water Quality Index (WQI) was calculated based on the formula developed by the Department of Environmental, Malaysia (DOE 2007).

Macroinvertebrates sampling was done on Pauh River bank. Surber net was used for macroinvertebrates sampling. Larger debris such as leaves, twigs, rocks and plants were removed and the macroinvertebrates organisms were kept using zipper plastic bag. The procedure had been repeated 10 times at the same station with different locations of the site. Samples were preserved with $70 \%$ ethanol and kept in fridge as specimen. Five bio-indices i.e Shannon Diversity Index, Margalef Diversity index and Pielou equality index, Average Score per Taxon (ASPT) and biological monitoring working party (BMWP) were selected in this study to describe the distribution of the communities of species. The Shannon Diversity Index, Margalef Diversity Index and Pielou Equality Index were obtained based on Eq. 3, Eq. 4 and Eq. 5. The ASPT (Average Score Per Taxon) is the sample BMWP score which is described in the Number of Families section, divided by the number of scoring families contributed in BMWP score.

Shannon Diversity Index

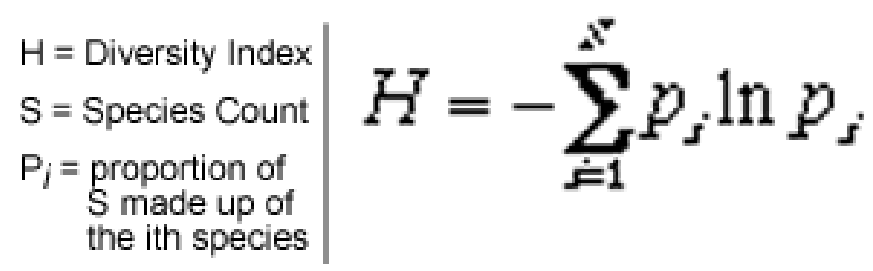

Eq. 3

Margalef Diversity Index

$D_{M g}=\frac{(S-1)}{\ln N}$

Eq. 4

$\mathrm{N}=$ the total number of individuals in the sample

$\mathrm{S}=$ the number of species recorded. 
Pielou Equality Index

$J^{\prime}=\frac{H^{\prime}}{H_{\max }^{\prime}}$

Eq. 5

$H^{\prime}$ is the number derived from the Shannon diversity index

$H_{\max }^{\prime}$ is the maximum possible value of $H^{\prime}$

\subsection{Statistical Analysis}

Principal component analysis was applied to seek the least number of factors which could account for common variance of a set of variables such as water pollutions and its' sources. Scree plot was used in this study to identify the number of extracted components. The plot is initially curved and then developed into a linear relationship at which the curve straightens out and these are taken as the maximum number of components to be extracted (Cattell1966).

Varimax rotation with Kaiser's normalization was applied to produce a new set of component. Each rotated component loading for every variable is differentiated significantly to identify each variable with a single component. This loading can be interpreted as a standardized regression coefficient, regressing the component on the measures (Decoster 1998). In deriving the component loadings, Burt-bank formula was used to calculate the significant level of loading. This formula has the merit of allowing not only for the sample size but also for the number of tests correlated and the number of components extracted up to and including the one under examination (Child 1970: 46). The following is the calculation of significant value of loading:

Standard error of a loading $=$ Standard error of a correlation $\sqrt{ }(n /(n+1-r))$

$$
\begin{aligned}
& =0.338 \sqrt{ } 26 /(26+1-3) \\
& =0.3518
\end{aligned}
$$

Whereas, Standard error of a correlation $=0.338$ at 0.05 significant level

$r=$ number of extracted factors

$\mathrm{n}=$ number of variables

Based on the eq. (1), component loading with \pm 0.3518 can be considered as a significant variable at 0.05 levels. The mathematical and statistical computations in this study were performed using Microsoft Excel 2013 and SPSS 16.0 software.

\section{Results and Discussion}

4.1 Water Quality Index (WQI) 


\section{Macrothink}

Environmental Management and Sustainable Development

ISSN 2164-7682

2016, Vol. 5, No. 1

In order to determine Pauh River Water Quality Index (WQI) based on Eq. 2 (DOE 2008), six parameters were chosen for this study namely $\mathrm{pH}$, dissolved oxygen (DO), chemical oxygen demand (COD), biological oxygen demand (BOD), Total suspended solid (TSS) and ammoniacal-nitrogen $\left(\mathrm{NH}_{3}-\mathrm{N}\right)$. Table 2 shows the analysis results for 6 stations of the parameters measured. By comparing to National Water Quality Standards for Malaysia (NWQS) (Table 3), DO, pH and TSS at 6 monitoring station were in Class II. BOD, COD and $\mathrm{NH}_{3}-\mathrm{N}$ in Station 6 was recorded in Class III, which was slightly polluted. As shown in Figure 2, water quality class II (Station 1-5) requires conventional treatment and suitable for sensitive aquatic species and recreational activities that involve bodily contact. Water quality class III (Station 6) requires extensive treatment and suitable for fishery common of economic value and tolerant species as well as source of drinking water for livestock.

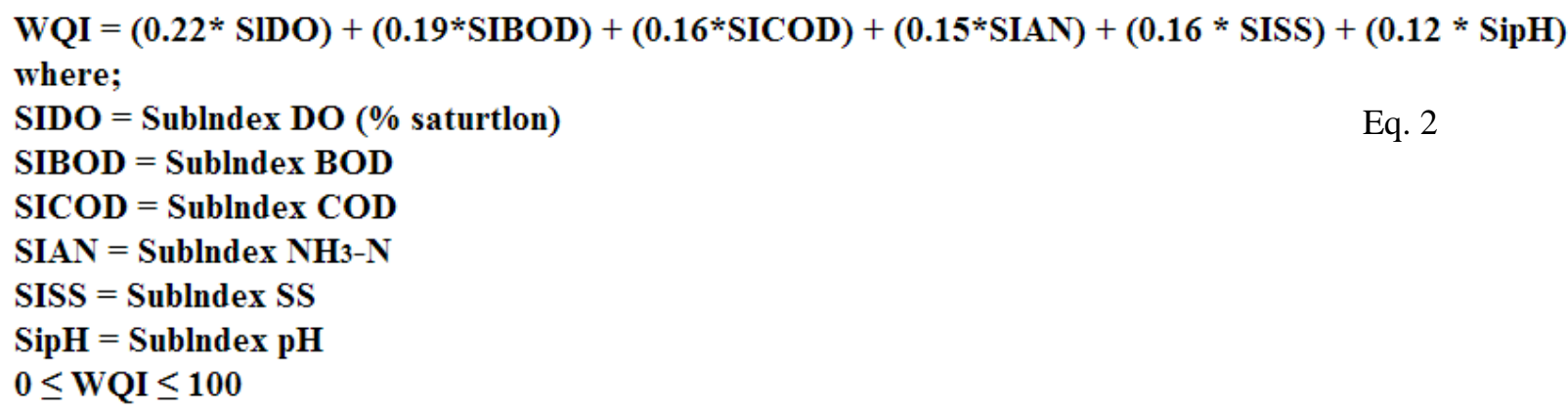

Table 2. Average of Six WQI parameters for Pauh River, Cameron Highlands.

\begin{tabular}{|l|l|l|l|l|l|l|}
\hline Parameters & Station 1 & Station 2 & Station 3 & Station 4 & Station 5 & Station 6 \\
\hline $\mathrm{DO}(\mathrm{mg} / \mathrm{l})$ & 7.14 & 7.09 & 6.98 & 7.11 & 7.13 & 5.60 \\
\hline $\mathrm{COD}(\mathrm{mg} / \mathrm{l})$ & 16.11 & 22.71 & 22.52 & 20.43 & 35.94 & 46.81 \\
\hline $\mathrm{BOD}(\mathrm{mg} / \mathrm{l})$ & 2.17 & 2.16 & 2.47 & 3.07 & 2.43 & 5.10 \\
\hline $\mathrm{NH}_{3}-\mathrm{N}(\mathrm{mg} / \mathrm{l})$ & 0.13 & 0.12 & 0.12 & 0.12 & 0.15 & 0.75 \\
\hline $\mathrm{pH}$ & 6.10 & 6.22 & 6.20 & 6.20 & 6.28 & 6.14 \\
\hline TSS $(\mathrm{mg} / \mathrm{l})$ & 0.20 & 0.02 & 0.03 & 0.01 & 0.02 & 0.03 \\
\hline
\end{tabular}

Table 3. National Water Quality Standards for Malaysia

\begin{tabular}{|l|c|c|c|c|c|c|}
\hline \multirow{2}{*}{\multicolumn{1}{c|}{ PARAMETER }} & \multirow{2}{*}{ UNIT } & \multicolumn{5}{c|}{ CLASS } \\
\cline { 3 - 7 } & & I & II & III & IV & V \\
\hline Ammoniacal Nitrogen & $\mathrm{mg} / \mathrm{l}$ & $<0.1$ & $0.1-0.3$ & $0.3-0.9$ & $0.9-2.7$ & $>2.7$ \\
\hline Biochemical Oxygen Demand & $\mathrm{mg} / \mathrm{l}$ & $<1$ & $1-3$ & $3-6$ & $6-12$ & $>12$ \\
\hline Chemical Oxygen Demand & $\mathrm{mg} / 1$ & $<10$ & $10-25$ & $25-50$ & $50-100$ & $>100$ \\
\hline Dissolved Oxygen & $\mathrm{mg} / \mathrm{l}$ & $>7$ & $5-7$ & $3-5$ & $1-3$ & $<1$ \\
\hline $\mathrm{pH}$ & - & $>7$ & $6-7$ & $5-6$ & $<5$ & $>5$ \\
\hline
\end{tabular}




\begin{tabular}{|c|c|c|c|c|c|c|c|}
\hline \multicolumn{2}{|c|}{ Total Suspended Solid } & $\mathrm{mg} / \mathrm{l}$ & $<25$ & $25-50$ & $50-150$ & $150-300$ & $>300$ \\
\hline \multicolumn{2}{|c|}{ Water Quality Index (WQI) } & - & $<92.7$ & $76.5-92.7$ & $51.9-76.5$ & $31.0-51.9$ & $>31$ \\
\hline Class I & $\begin{array}{l}\text { Conservation of na } \\
\text { Water Supply I - P } \\
\text { Fishery I - Very se }\end{array}$ & $\begin{array}{l}\text { ral ens } \\
\text { tically } \\
\text { tive ac }\end{array}$ & $\begin{array}{l}\text { ironmen } \\
\text { no treat } \\
\text { uatic sp }\end{array}$ & $\begin{array}{l}\text { t. } \\
\text { ment necess } \\
\text { ecies. }\end{array}$ & ary. & & \\
\hline Class IIA & \multicolumn{7}{|c|}{ Water Supply II - Conventional treatment. } \\
\hline Class IIB & \multicolumn{7}{|c|}{ Recreational use body contact. } \\
\hline Class III & Fishery III - Comr & $\begin{array}{l}\text { Vater } S \\
\text { n of ec }\end{array}$ & $\begin{array}{l}\text { upply II } \\
\text { onomic } \\
\text { livesto }\end{array}$ & $\begin{array}{l}\text { I - Extensive } \\
\text { value and to } \\
\text { ck drinking. }\end{array}$ & $\begin{array}{l}\text { treatment } \mathrm{r} \\
\text { lerant specie }\end{array}$ & $\begin{array}{l}\text { equired. } \\
\text { es; }\end{array}$ & \\
\hline $\begin{array}{l}\text { Class IV } \\
\text { Class V }\end{array}$ & $\begin{array}{l}\text { Irrigation } \\
\text { None of the above }\end{array}$ & & & & & & \\
\hline
\end{tabular}

(Source: DOE, 2007)

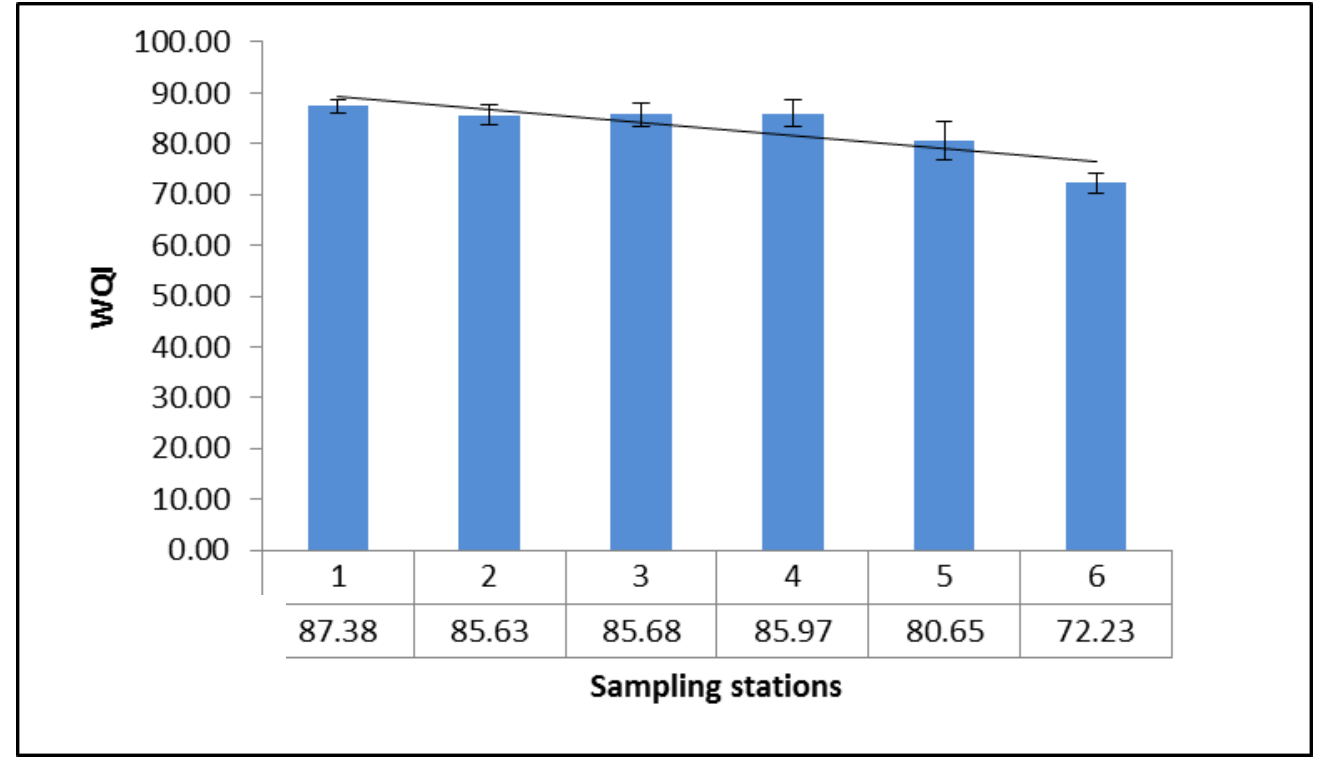

Figure 2. Average of Water Quality Index (WQI) for six monitoring stations

\subsection{Distribution of Benthic Macroinvertebrates by Location (Spatial)}

Table 4 shows the abundance of macroinvertebrates expresses as percentage of total, found from each sampling period. The macroinvertebrates found were from the groups of Coleoptera, Trichoptera, Hemiptera, Ephemeroptera, Plecoptera, Diptera, Odonata, Gastropoda, Hirudinea and Oligochaeta. A total of 51 families were identified from the sampling sites during the sampling period. The lack of obvious difference in the presence or absence of the macroinvertebrates during the alternate monthly samplings indicated Pauh River was not fluctuating and a total $90 \%$ of the macroinvertebrates could be consistently found in the sampling site.

Table 4. Macroinvertebrates abundance, as percentage (expressed of total in a month period) 
found at the Pauh River, Cameron Highlands

\begin{tabular}{|c|c|c|c|c|c|c|c|c|}
\hline \multirow[b]{2}{*}{ Phylum } & \multirow[b]{2}{*}{ Class } & \multirow[b]{2}{*}{ Order } & \multirow[b]{2}{*}{ Family } & \multicolumn{5}{|c|}{ ind/m2 } \\
\hline & & & & Feb & Apr & Jun & Aug & Oct \\
\hline \multirow[t]{7}{*}{ Arthropoda } & Insecta & Coleoptera & Elmidae & 0.88 & 3.75 & 17.71 & 16.98 & 26.56 \\
\hline & & & Psephenidae & 0.75 & 0.94 & 1.25 & 2.40 & 4.58 \\
\hline & & & Sperchidae & 0.75 & 0.21 & 0.63 & 0.83 & 1.56 \\
\hline & & & Hydrophilidae & 0.13 & 3.65 & 1.98 & 1.04 & 1.04 \\
\hline & & & Dryopidae & 2.38 & 1.35 & 1.56 & 4.48 & 0.21 \\
\hline & & & Eulichalidae & 0.25 & 0.31 & 0.31 & 0.21 & 0.42 \\
\hline & & & Scirtidae & - & 0.31 & 0.42 & 2.60 & 0.83 \\
\hline \multirow[t]{2}{*}{ Arthropoda } & Insecta & Hemiptera & Aphelocheiridae & 1.63 & 1.46 & 3.23 & 1.77 & 3.65 \\
\hline & & & Mesoveliidae & 0.88 & 0.42 & 0.21 & 0.73 & 0.31 \\
\hline \multirow[t]{3}{*}{ Arthropoda } & Insecta & Plecoptera & Perlidae & 12.88 & 23.44 & 25.31 & 32.92 & 46.98 \\
\hline & & & Peltoperlidae & 0.63 & 0.31 & 0.42 & 0.52 & 0.52 \\
\hline & & & Nemouridae & 3.25 & 3.54 & 5.21 & 3.75 & 8.54 \\
\hline \multirow[t]{3}{*}{ Arthropoda } & Insecta & Ephmeroptera & Baetidae & 2.13 & 25.21 & 32.50 & 25.42 & 30.31 \\
\hline & & & Heptageniidae & 3.25 & 8.65 & 10.31 & 8.23 & 10.00 \\
\hline & & & Leptophlebiidae & - & 1.56 & 4.48 & 4.90 & 2.50 \\
\hline \multirow[t]{5}{*}{ Arthropoda } & Insecta & Trichoptera & Hydropsychidae & 4.25 & 14.27 & 19.58 & 16.46 & 23.02 \\
\hline & & & Leptoceridae & 1.00 & 5.73 & 1.04 & 0.42 & 8.54 \\
\hline & & & Lepistomadidae & 3.50 & 4.48 & 7.40 & 10.42 & - \\
\hline & & & Polycentropodidae & 0.50 & 2.81 & 3.33 & 4.27 & 3.44 \\
\hline & & & Philopotamidae & 0.38 & 1.56 & 4.17 & 7.60 & 1.25 \\
\hline \multirow[t]{2}{*}{ Arthropoda } & Insecta & Odonata & Libellulidae & 1.13 & 1.67 & 0.63 & 1.35 & 1.46 \\
\hline & & & Gomphidae & 0.63 & - & - & - & \\
\hline \multirow[t]{5}{*}{ Arthropoda } & Insecta & Diptera & Tipulidae & 0.63 & 1.04 & 3.44 & 2.19 & 4.90 \\
\hline & & & Simuliidae & 10.25 & 28.02 & 22.81 & 63.54 & 63.44 \\
\hline & & & Chironomidae & 16.38 & 60.52 & 484.48 & 409.69 & $\begin{array}{l}256.9 \\
8\end{array}$ \\
\hline & & & Ceraptopogonidae & - & 1.56 & 3.13 & 3.33 & 1.25 \\
\hline & & & Athericidae & - & 0.10 & 0.10 & 0.73 & 0.21 \\
\hline Arthropoda & $\begin{array}{l}\text { Malacostra } \\
\text { ca }\end{array}$ & Decapoda & Sesarmidae & 0.50 & 0.31 & 0.31 & 0.42 & 0.63 \\
\hline \multirow[t]{2}{*}{ Mollusca } & Gastropoda & Neogastropod & Planorbidae & - & - & 1.46 & 1.15 & 0.21 \\
\hline & & & Physidae & 0.13 & 20.42 & 27.40 & 9.58 & 10.42 \\
\hline \multirow[t]{3}{*}{ Annelida } & Oligochaeta & Opisthopora & Tubificidae & - & 0.10 & 19.69 & 3.85 & 59.90 \\
\hline & & & Lumbriculidae & - & 0.31 & 0.42 & 0.63 & 1.04 \\
\hline & & & Naididae & - & 0.10 & - & 0.21 & 0.31 \\
\hline Annelida & Hirudinea & Gnathobdellida & Erpobdellidae & - & 1.67 & 6.77 & 2.08 & 9.48 \\
\hline Platyheminthes & Turbellaria & Seriata & Dugesiidae & - & 1.35 & 1.46 & 0.63 & - \\
\hline
\end{tabular}

Table 5 shows the distribution and average density of phylogenetic benthic macroinvertebrate 
fauna for each monitoring station according to Phylum, Class, Order and Family. A total 28 to 31 taxa have been found in Station 1, 2, 3 and 5 (upstream to middle stream). However, only 7 taxa found at station 6 (downstream). Total 31 taxa with an average density $368.28 \mathrm{ind} / \mathrm{m}^{2}$ were found in Station 4 which was highest number of taxa among the monitoring stations. According to WQI analysis result, Station 4 has been recorded as Class II, which is suitable for sensitive aquatic species. The may be the reason abundance of macroinvertebrates found in this location. Only 7 taxa were identified in Station 6 which was the lowest number of taxa among monitoring stations; however it recorded highest density of phylogenetic benthic macroinvertebrate fauna $\left(677.77 \mathrm{ind} / \mathrm{m}^{2}\right)$. It was contributed by Chironomidae $(466.75$ $\left.\mathrm{ind} / \mathrm{m}^{2}\right)$, Planorbidae $\left(3.38 \mathrm{ind} / \mathrm{m}^{2}\right)$, Erpobdellidae $\left(24.0 \mathrm{ind} / \mathrm{m}^{2}\right)$, Tubificidae $\left(99.63 \mathrm{ind} / \mathrm{m}^{2}\right)$, Baetidae $\left(2.38 \mathrm{ind} / \mathrm{m}^{2}\right)$, Lumbriculidae $\left(0.25 \mathrm{ind} / \mathrm{m}^{2}\right)$ and Physidae $\left(81.38 \mathrm{ind} / \mathrm{m}^{2}\right)$.

Furthermore, the abundance of Diptera; Chironomiidae and Simuliidae in Pauh river indicates organic pollution (Buckup et al. 2007; Kusza 2005) due to the recreational function of the river. The river is polluted with domestic waste throw by the nearby recreational activities especially foods waste and detergent used to wash off their culinary sets.

Overall, the Shannon Diversity Index, Margalef Diversity Index, Pielou Equality Index, Average Score per Taxon (ASPT) and Biological Monitoring Working Party (BMWP) have been decreased from station 1 to station 6. As mentioned, it was due to decrease of water quality index from Class II to Class III. Table 6 shows the average value of biological index according to monitoring stations.

Table 5. Distribution and average density of phylogenetic macroinvertebrate fauna

\begin{tabular}{|c|c|c|c|c|c|c|c|c|c|}
\hline \multirow[b]{2}{*}{ Phylum } & \multirow[b]{2}{*}{ Class } & \multirow[b]{2}{*}{ Order } & \multirow[b]{2}{*}{ Family } & \multicolumn{6}{|c|}{$\left(\mathrm{ind} / \mathrm{m}^{2}\right)$} \\
\hline & & & & $\begin{array}{c}\text { Statio } \\
\text { n } 1\end{array}$ & $\begin{array}{c}\text { Statio } \\
\text { n } 2\end{array}$ & $\begin{array}{c}\text { Statio } \\
\text { n } 3\end{array}$ & $\begin{array}{c}\text { Statio } \\
\text { n } 4\end{array}$ & $\begin{array}{c}\text { Statio } \\
\text { n } 5\end{array}$ & $\begin{array}{c}\text { Statio } \\
\text { n } 6\end{array}$ \\
\hline \multirow[t]{7}{*}{ Arthropoda } & Insecta & Coleoptera & Elmidae & 7.71 & 7.81 & 8.13 & 19.48 & 22.61 & - \\
\hline & & & Psephenidae & 4.06 & 2.19 & 1.98 & 0.73 & 0.83 & - \\
\hline & & & Sperchidae & 1.15 & 0.73 & 0.938 & 0.83 & 0.31 & - \\
\hline & & & Hydrophilidae & 1.25 & 1.36 & 1.56 & 1.15 & 2.50 & - \\
\hline & & & Dryopidae & 5.73 & 1.36 & 1.56 & 1.15 & 2.50 & - \\
\hline & & & Eulichalidae & 0.21 & - & 0.52 & 0.52 & 0.21 & - \\
\hline & & & Scirtidae & 0.73 & 0.32 & 0.21 & 1.36 & 1.56 & - \\
\hline \multirow[t]{2}{*}{ Arthropoda } & Insecta & Hemiptera & Aphelocheiridae & 1.98 & 0.73 & 1.36 & 5.94 & 1.46 & - \\
\hline & & & Mesoveliidae & 0.52 & 0.42 & 0.63 & 0.83 & 0.11 & - \\
\hline \multirow[t]{3}{*}{ Arthropoda } & Insecta & Plecopetera & Perlidae & 38.23 & 38.86 & 26.88 & 19.38 & 12.08 & - \\
\hline & & & Peltoperlidae & 0.94 & 0.21 & 0.11 & 0.94 & 0.11 & - \\
\hline & & & Nemouridae & 3.86 & 2.29 & 3.65 & 6.98 & 6.98 & - \\
\hline \multirow[t]{3}{*}{ Arthropoda } & Insecta & Ephemeroptera & Baetidae & 18.86 & 27.08 & 28.33 & 28.86 & 10.11 & 2.38 \\
\hline & & & Heptageniidae & 10.63 & 11.77 & 10.42 & 3.65 & 3.02 & - \\
\hline & & & Leptophlebiidae & 5.11 & 2.40 & 3.02 & 2.71 & 0.42 & - \\
\hline
\end{tabular}




\begin{tabular}{|c|c|c|c|c|c|c|c|c|c|}
\hline Arthropoda & Insecta & Trichopetra & Hydropsychidae & 12.08 & 5.31 & 13.86 & 22.81 & 22.81 & - \\
\hline & & & Leptoceridae & 7.40 & 4.38 & 1.56 & 2.92 & 0.31 & - \\
\hline & & & Lepistomadidae & 6.77 & 7.08 & 1.25 & 1.67 & 5.52 & - \\
\hline & & & $\begin{array}{l}\text { Polycentropodid } \\
\text { ae }\end{array}$ & 5.00 & 2.61 & 4.90 & 1.04 & 0.73 & - \\
\hline & & & Philopotamidae & 8.65 & 4.48 & 0.73 & 0.52 & 0.52 & - \\
\hline \multirow[t]{2}{*}{ Arthropoda } & Insecta & Odonata & Libellulidae & 0.83 & 1.56 & 2.40 & 0.94 & 0.63 & - \\
\hline & & & Gomphidae & 0.42 & 0.11 & - & - & - & - \\
\hline \multirow[t]{6}{*}{ Arthropoda } & Insecta & Diptera & Tipulidae & 4.58 & 1.88 & 1.46 & 1.98 & 2.19 & - \\
\hline & & & Simuliidae & 48.54 & 66.67 & 56.36 & 4.69 & 4.27 & - \\
\hline & & & & & & 206.9 & 231.0 & 230.5 & 466.7 \\
\hline & & & Chironomidae & 51.25 & 68.65 & 8 & 4 & 2 & 5 \\
\hline & & & $\begin{array}{l}\text { Ceraptopogonid } \\
\text { ae }\end{array}$ & 1.67 & 2.08 & 1.25 & 2.71 & 1.88 & - \\
\hline & & & Athericidae & 0.21 & 0.11 & 0.42 & 0.42 & - & - \\
\hline Arthropoda & $\begin{array}{l}\text { Malacostra } \\
\text { ca }\end{array}$ & Decapoda & Sesarmidae & 0.83 & 0.11 & 0.42 & 0.42 & - & - \\
\hline \multirow[t]{2}{*}{ Mollusca } & Gastropoda & Neogastropod & Planorbidae & - & - & - & - & - & 3.38 \\
\hline & & & Physidae & - & - & - & - & - & 81.38 \\
\hline \multirow[t]{3}{*}{ Annelida } & $\begin{array}{l}\text { Oligochaet } \\
\text { a }\end{array}$ & Opisthopora & Tubificidae & - & - & - & 0.42 & 0.11 & 99.63 \\
\hline & & & Lumbriculidae & - & - & 0.94 & 0.73 & 0.52 & 0.25 \\
\hline & & & Naididae & 0.31 & 0.11 & - & 0.21 & - & - \\
\hline Annelida & Hirudinea & Gnathobdellida & Erpobdellidae & - & - & - & - & - & 24 \\
\hline Platyhelminthes & Turbellaria & Seriata & Dugesiidae & - & 0.31 & 1.88 & 1.25 & 0.83 & - \\
\hline
\end{tabular}

Note: “ - " indicated zero density of phylogenetic macroinvertebrate fauna

Table 6. Selected Bio-indices for 6 sampling stations in Pauh River, Cameron Highlands

\begin{tabular}{|l|l|l|l|l|l|l|}
\hline Index & Station 1 & Station 2 & Station 3 & Station 4 & Station 5 & Station 6 \\
\hline Shannon & $2.39 \pm 0.03$ & $2.06 \pm 0.17$ & $1.79 \pm 0.47$ & $1.70 \pm 0.35$ & $1.37 \pm 0.35$ & $0.81 \pm 0.25$ \\
\hline Margalef & $3.76 \pm 0.19$ & $3.25 \pm 0.40$ & $3.13 \pm 0.28$ & $3.45 \pm 0.16$ & $2.85 \pm 0.44$ & $0.70 \pm 0.13$ \\
\hline Pielou & $0.75 \pm 0.03$ & $0.69 \pm 0.06$ & $0.61 \pm 0.18$ & $0.55 \pm 0.13$ & $0.47 \pm 0.13$ & $0.46 \pm 0.13$ \\
\hline BMWP & $140.80 \pm 10.24$ & $127.60 \pm 12.34$ & $121.20 \pm 20.55$ & $138.00 \pm 8.63$ & $111.00 \pm 13.36$ & $13.00 \pm 3.67$ \\
\hline ASPT & $6.03 \pm 0.31$ & $6.54 \pm 0.59$ & $5.97 \pm 0.27$ & $6.07 \pm 0.24$ & $5.95 \pm 0.32$ & $2.25 \pm 0.56$ \\
\hline
\end{tabular}

\subsection{Principal Component Analysis (PCA)}

This study demonstrated the application of Principal component analysis in order to group different variables with similar characteristics. Principal component analysis (PCA) was 


\section{Macrothink}

performed on 26 variables to produce a model that explained the positive or negative relationship between (i) water quality physico-chemical parameters, (ii) characteristics of habitat and (iii) benthic macroinvertebrate families. Based on Eq. (1), component loadings with \pm 0.3518 was considered as a significant variable at 0.05 levels. There were three principal components (PCs) were extracted from 26 variables, which eigenvalues were greater than 2.0, as shown by scree plot (Figure 2). Hence, three components accounted for $72.15 \%$ of the total variance of the variables were used for this study. Table 7 showed the three components extracted from PCA.

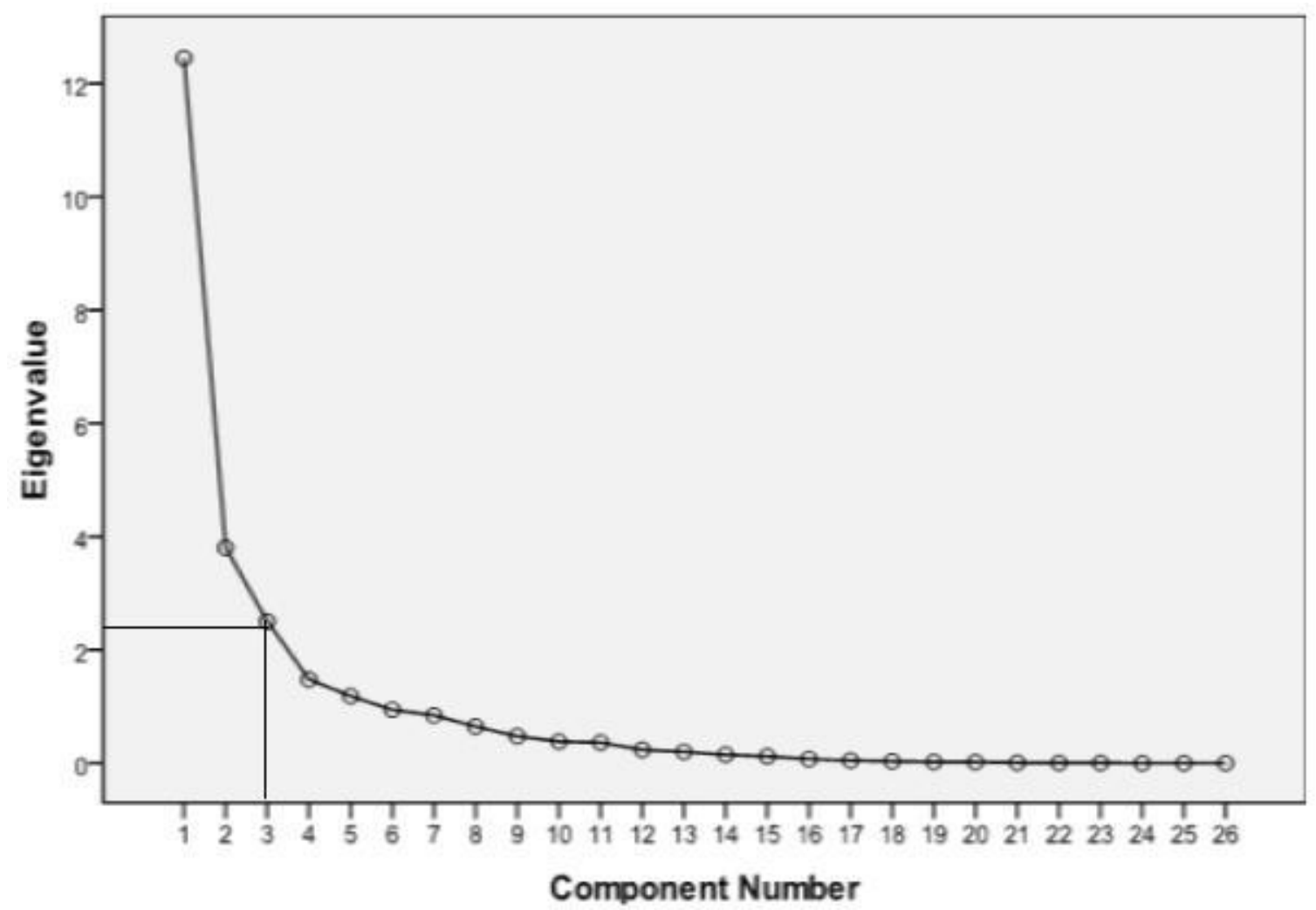

Figure 2. Scree Plot of Principal Components (PCs)

Table 7. Rotated loadings for the three extracted components

\begin{tabular}{|l|c|c|c|}
\hline Variables & PC 1 & PC 2 & PC3 \\
\hline$\underline{\text { i. Water quality parameters }}$ & & & \\
\hline Temperature & $\underline{0.567}$ & -0.346 & -0.168 \\
\hline Conductivity & $\underline{0.905}$ & -0.256 & -0.027 \\
\hline TDS & $\underline{0.901}$ & -0.228 & -0.153 \\
\hline DO & -0.306 & $\underline{0.390}$ & $\underline{0.696}$ \\
\hline $\mathrm{BOD}$ & $\underline{0.516}$ & -0.247 & -0.203 \\
\hline $\mathrm{NH}_{3}-\mathrm{N}$ & $\underline{0.843}$ & -0.258 & -0.161 \\
\hline Water Quality Index (WQI) & $\underline{-0.617}$ & $\underline{0.518}$ & 0.256 \\
\hline$\underline{\text { ii. Characteristics of habitat }}$ & $\underline{-0.432}$ & $\underline{0.551}$ & -0.136 \\
\hline & \multicolumn{3}{|l}{} \\
\hline
\end{tabular}




\begin{tabular}{|l|c|c|c|}
\hline Cobblestones & & & \\
\hline Clay & 0.221 & -0.221 & 0.259 \\
\hline Canopy & -0.268 & $\underline{0.703}$ & -0.318 \\
\hline River width & $\underline{0.902}$ & 0.031 & -0.268 \\
\hline River depth & $\underline{0.916}$ & -0.167 & -0.186 \\
\hline Stream flow & -0.067 & $-\underline{0.588}$ & $\underline{-0.366}$ \\
\hline iii. Families of benthic macro-invertebrate fauna & & & \\
\hline Elmidae & -0.244 & 0.030 & $\underline{0.866}$ \\
\hline Psephenidae & -0.015 & $\underline{0.738}$ & 0.344 \\
\hline Perlidae & -0.197 & $\underline{0.812}$ & 0.287 \\
\hline Nemouridae & -0.338 & -0.060 & $\underline{0.794}$ \\
\hline Heptageniidae & -0.264 & $\underline{0.798}$ & -0.087 \\
\hline Leptoceridae & 0.040 & 0.284 & 0.214 \\
\hline Polycentropodidae & -0.229 & $\underline{0.845}$ & -0.049 \\
\hline Simuliidae & -0.085 & $\underline{0.867}$ & 0.008 \\
\hline Chironomidae & 0.193 & -0.056 & 0.254 \\
\hline Planorbidae & $\underline{0.753}$ & -0.202 & -0.192 \\
\hline Hippeutis & $\underline{0.503}$ & -0.055 & -0.122 \\
\hline Tubificidae & $\underline{0.819}$ & -0.082 & -0.020 \\
\hline Erpobdellidae & $\underline{0.886}$ & -0.120 & -0.082 \\
\hline
\end{tabular}

\subsubsection{First Component (PC1)}

First component (PC1) was represented by benthic macroinvertebrates of Planorbidae (0.753), Hippeutis (0.503), Tubificidae (0.819) and Erpobdellidae (0.886). These benthic macroinvertebrates families are only available at the downstream monitoring stations e.g station 4,5 and 6 . There were positive relationship with temperature $(0.567)$, conductivity (0.905), TDS (0.091), BOD (0.516) and $\mathrm{NH}_{3}-\mathrm{N}(0.843)$, and negative relationship with Water Quality Index (WQI). Besides that, the density benthic macro-invertebrates families correlated with characteristics of river habitat i.e cobblestones (-0.432), depth (0.916) and width (0.902) of Pauh River. Hence, this component could be classified as benthic macroinvertebrates which tolerated to low water quality condition and high loading of organic matters.

Parameters of temperature, conductivity, TDS, $\mathrm{NH}_{3}-\mathrm{N}$ and Biological Oxygen Demand (BOD) at Station 6 were recorded highest value which indicated the high concentration of organic matters in the station. Station 6 is located at the downstream and receives higher concentration of organic matters such as decayed leaves and silt settlement along the Sungai Pauh including tributaries. Kucuk (2008) and Yap et al. (2006) state that density of benthic macroinvertebrates can will be affected by organic pollutants. Average WQI in Station 6 was recorded as 72.32 (Class III) which was slightly polluted based on Malaysian National Water Quality Standard. Based on PC1, the density of benthic macroinvertebrates families increased with higher Biological Oxygen Demand (BOD) concentration. The density of Planorbidae in station 6 is recorded $3.38 \mathrm{ind} / \mathrm{m}^{2}$. It is grouped under Neogastropod taxa and recognized as 
hydrophytic organisms which able undergoes self-fertilization and reproduction process at least once a year (Gooderham \& Tsyrlin 2003; Flores \& Zafaralla 2012). Furthermore, this family is able to survive in different aquatic habitats, preferably, at the lower stream flow $(<0.5 \mathrm{~m} / \mathrm{s})$ and high concentration of organic matters such as plankton, detritus and decayed leaves (Gooderham \& Tsyrlin 2003; Kucuk 2008; Flores \& Zafaralla 2012; Elexová \& Némethová 2003, Miserendino et al. 2008).

Tubificidae family is able to tolerate low oxygen conditions; even in the concentration of oxygen gas is approaching $0 \mathrm{mg} / \mathrm{L}$ (Yule \& Yong 2004; Gooderham \& Tsyrlin 2003). It often is used as indicators of aquatic habitats polluted organic environment (Lobo \& Alves 2011; Gooderham \& Tsyrlin 2003). In this study, the Tubificidae family was found in downstream stations (Station 4, 5 and 6). However, the density of Tubificidae family was found as 99.63 in Station 6 where recorded as slightly polluted (Class III). High concentration of organic material, moderate stream flow and mud provide suitable habitat for Tubificidae family (Lafront 1984, Kucuk 2008; Matins et al. 2008). Sauter \& Gude (1996) show that positive correlation between the density Tubificidae and fine sediment fraction $(<0: 21 \mathrm{~mm})$. Besides, Moore (1979) emphasizes the importance of organic matter on the distribution of benthic macroinvertebrates. Presence of organic material increase the concentration of algae which becomes food source for Tubificidae (Lobo and Alves 2011)

Erpobdellidae is grouped under Hirudinea taxa. The Erpobdellidae was only found in Station 6 which density as $24 \mathrm{ind} / \mathrm{m}^{2}$. Erpobdellidae family is highly tolerance at low water quality condition and low dissolved oxygen (Pliūraitè and Mickéniené 2009; Paisley et al. 2003; Muñoz and Prat 1996). According to Marques et al. (1999), Erpobdellidae does not have specific habitat requirements but tends high organic loading and low oxygen levels (Miserendino et al. 2008).

\subsubsection{Second Component (PC2)}

Second component (PC2) was represented by Psephenidae (0.738), Perlidae (0.815), Heptageniidae (0.798), Polycentropodidae (0.845) and Simulidae (0.867), WQI (0.51) and dissolved oxygen (DO) (0.390). The benthic macro-invertebrates families loaded in this component are sensitive to water environment such as $\mathrm{NH}_{3}-\mathrm{N}$, dissolved oxygen (DO), organic matter and stream flow. There were significant relationship between benthic macro-invertebrates families and characteristics of river habitat i.e cobblestones $(0.551)$ and canopy (0.703) and inversely relationship with the stream flow (-0.588).

River bed composition is recognized to affect a significant impact on the biomass and diversity of benthic macroinvertebrates (Flores \& Zafaralla 2012). The characteristics of river are essential to the survival and reproduction of benthic macroinvertebrates as well as supporting their respiratory process (Lobo \& Alves 2011). Muñoz \& Prat (1996) state that density of benthic macroinvertebrates families will be decreased in polluted areas. Comparison of benthic macroinveterbrates families in this study at upstream and downstream stations indicate that was affected significantly as water quality conditions.

Dynamic stream flow plays an important role in the distribution of small benthic 
macroinvertebrates. According to Ramirez and Pringle (1998), the density and richness of benthic macro-invertebrate taxa are mainly based on the stream flow. This may due to the higher stream flow increases the concentration of dissolved oxygen (Moore 1979) and food for some benthic macro-invertebrate families e.g Trichoptera (Flores \& Zafaralla 2012). However, the stream flow recorded in this study has a negative relationship with the density of benthic macro-invertebrate families. It may be flushed to downstream when the Pauh River's stream flow is high during raining season.

Perlidae and Peltoperlidae under Plecoptera taxa are recognized as sensitive to water pollution. They are usually found in a river which contains high oxygen concentration (Hynes 1970; Pliūraitė and Mickènienė 2009; Kato et al. 2005). The PCA result shows significant correlation among Perlidae, dissolved oxygen (DO) and WQI, whereas higher DO increases the density Perlidae family in Pauh River. Besides, temperature is inversely proportional to the concentration of dissolved oxygen (DO). Hence, higher temperature may reduce the dissolved oxygen concentration in Pauh River.

Based on PC2, population of Heptageniidae families are highly affected by dissolved oxygen (DO) and river habitat characteristic e.g canopy. The density of Heptageniidae increases (density from 3.02 to $10.63 \mathrm{ind} / \mathrm{m}^{2}$ ) from monitoring station 6 to 1 . The density of Heptageniidae increases with high oxygen concentration and low water temperature. Besides, habitat characteristic of canopy produces low temperature of water environment which suitable for Heptageniidae family.

Polycentropodidae larvae are sensitive to water quality conditions e.g low temperature and high oxygen concentration (Edmunds \& Waltz 1996). Trichopetra taxa widely used to assess the health of the river (Muñoz and Prat 1996; Gooderham \& Tsyrlin 2003). In this study, Polycentropodidae larvae were found only in monitoring station 1 to station 5 . The water quality statuses in these monitoring stations were classified as Class I and II based on Malaysian National Water Quality Standard. Based on the characteristics of habitat in these stations, rocky substrates were found which provided an ideal habitat to Trichoptera taxa. According to Maul et al. (2004), Trichoptera and Emphemeroptera taxa can be found in river containing low concentrations of phosphorus. Griffith et al. (2001) state Ephemeroptera taxa are sensitive to nutrient loading. At low nutrients loading level, population of Polycentropodidae may be recorded as high density (Miserendino et al. 2008). Although, Phosphorus concentration was not measured in this study, the same scenario may be occurred in Pauh River.

Simuliide larvae usually is found in the areas where rich of nutrients (Gooderham \& Tsyrlin 2003; Camargo 1992; Loch et al. 1996). Simuliida larvae are abundant in the mountainous lake which rich of nutrient (Mohsen \& Mulla 1982). Mohsen \& Mulla (1982) states that Simuliidae larvae found in the moderate stream flow $(0.6 \mathrm{~m} / \mathrm{s})$. Besides, aquatic plants in the river banks provide shelter to Simuliidae larvae and increase the population (Pachón \& Walton 2011). However, ammonia-nitrogen substance $\left(\mathrm{NH}_{3}-\mathrm{N}\right)$ is harmful to Simuliidae larvae. Pachón \& Walton (2011) identifies that $0.6 \mathrm{mg} / \mathrm{L}$ of $\mathrm{NH}_{3}-\mathrm{N}$ is able to kill $100 \%$ of Simuliidae larvae within 4 hours. Hence, Simuliidae larvae were not found in Station 6 which 
was recorded as $0.75 \mathrm{mg} / \mathrm{L}$ of $\mathrm{NH}_{3}-\mathrm{N}$. The density of Simuliidae was decreased from upstream to downstream (from 48.54 to $0 \mathrm{ind} / \mathrm{m}^{2}$ ) which indicated the degradation of water quality at Pauh River downstream. Besides, this study identifies the negative correlation between the stream flow in Pauh River and Simuliidae families. Tietze and Mulla (1989) emphases that Simuliidae larvae prefer living in moderate stream flow environment with aquatic plants on the banks (Howitt, 2008).

\subsubsection{Third Component(PC3)}

Third component (PC3) was represented by Nemouridae (0.794) and Elmidae (0.866), stream flow (-0.366) and dissolved oxygen (DO) (0.696). The benthic macroinvertebrate families loaded in this component are recognized as species which do not tolerate low concentration of dissolved oxygen. According to Grandjean et al. (2003), Nemouridae family is the most sensitive Plecoptera taxa. It normally colonizes in the low water temperature and high dissolved oxygen (Zwick 2004). Nempouridae is identified as detritus eaters (Grandjean et al. 2003; Harper 1973), hence the presence of decomposed leaves, aquatic plants and other aquatic organisms in stations 1 and 5 supports the population of Nempouridae family. However, Nemouridae families are not able to tolerate water pollution (Zwick 2004). Blackburn and Mazzacano (2012) mention that existence of Nemouridae families is affected by stream flow of the river. In this study, density of Nemouridae families was inversely proportional to Pauh River's stream flow, which further concluded that this family could not tolerate to the stream flow greater than $1.0 \mathrm{~m} / \mathrm{s}$. However, this study was not able to identify the Nemouridae families at the Station 6 where the stream flow was $0.3 \mathrm{~m} / \mathrm{s}$ as the location was slightly polluted.

Elmidae families are used as biological indicator to monitor water quality. The density of Elmidae was found in this It is usually found in the upper reaches of the river where contains have high concentration of DO (Gooderham \& Tsyrlin 2003; Kohut 2007). There was positive relationship between Elmidae families with the concentration of DO. Elmidae families are also affected by domestic pollutants e.g soaps and detergents (Elliot, 2008). The wetting agent of soaps and detergents can destroy the plastron which is essential for the respiration of Elmidae (Elliot 2008). Besides, PC3 showed that the density of Nemouridae families was inversely proportional to Pauh River's stream flow. Hence, this study further concludes that Nemouridae families prefer to live in the stream flow environment (does not greater than $0.5 \mathrm{~m} / \mathrm{s}$ ) with filamentous algae. Higher stream flow may devastate the Elmidae's habitat as it will be embedded in the roots of aquatic plants or space between cobblestones (Elliot 2008; Elliot 2006). However, due to water pollution, this study was not able to identify the Nemouridae families at the Station 6 where the stream flow was recorded as $0.3 \mathrm{~m} / \mathrm{s}$.

\section{Conclusion}

This study demonstrated the application of PCA to evaluate the relationship among water quality parameters, benthic macroinvetebrate organism and its habitat characteristic. The outcome showed that there were number of potential benthic macroinvetebrates could be used to monitor water quality condition in highlands region. Besides being used in the monitoring of the environment, macroinvetebrate were suggested to be used as biological monitoring in 
recovery and conservation efforts in the future. Further study is also proposed to identify the relationship of benthic macroinvetebrate organism, heavy metals, and biological parameters such as e-coliform and fecal coliform which are important for the comprehensive river water quality monitoring tasks.

\section{Reference}

APHA. (1992). Standard Methods for Examination of Water and Wastewater, 18th ed., American Public Health Association (APHA), AWWA \& WPCF, Washington D. C.

APHA. (1998). Standard Methods for Study of Examination of Water and Wastewater, 20th ed., American Public Health Association (APHA), AWWA \& WPCF, Washington.

Azrina MZ, Yap CK, Ismail AR, Ismail A., \& Tan S. G. (2006). Anthropogenic impacts on the distribution and biodiversity of benthic macroinvertebrates and water quality of the Langat River, Peninsular Malaysia. Ecotoxicol Environ. Saf .64, 337-347. http://dx.doi.org/10.1016/j.ecoenv.2005.04.003

Blackburn, M., \& Mazzacano, C. (2012). Using aquatic macroinvertebrates as indicators of streamflow duration. USA: The Xerces Society for Invertebrate Conservation.

Buckup, L., Bueno, A. A. P., Bond-Buckup, G., Casagrande, M., \& Majolo, F. (2007). The benthic fauna of highland stream in southern Brazil: composition, diversity and structure. $\begin{array}{llll}\text { Revista Brasileira } \quad \text { de 29ologica } & \text { 24(2), }\end{array}$ http://dx.doi.org/10.1590/S0101-81752007000200005

Camargo, J. A. (1992). Temporal and spatial variations in dominance, diversity and biotic indices along a limestone stream receiving a trout farm effluent. Water Air Soil Pollut. 63, 343-359. http://dx.doi.org/10.1007/BF00475501

Cattell, R. B. (1966). Handbook of multivariate experimental psychology. Rand McNally Chicago,

Child D. (1979). The essentials of factor analysis. Hol Rienheart and Winston, London.

DeCoster J. (1998). Overview of factor analysis. University of Alabama.

Department of Environment, DOE. (2007). Malaysia Environmental Quality Report 2006, In : Chapter 3: River Water Quality. Putrajaya.

Department of Environment, DOE (2008). Malaysia Environmental Quality Report 2008. Department of Environment, Ministry of Natural Resources and Environment Malaysia, Putrajaya.

Department of Survey and Mapping (JUPEM). (2012). Map of Pahang State. 1:250,000. Kuantan, Malaysia.

Dinakaran, S., \& Anbalagan, S. (2007). Antropogenic impacts on aquatic insects in six streams of south Western Ghats. Journal of Science 7 (37): 1536-2442.

Edmunds, J. G. F. \& Waltz, R. D. (1996). An introduction to the Aquatic Insects of North 
America. Edisi ke-6.

Einax J.W., Truckenbort D., Kampe O. (1998). River pollution data interpreted by means of $\begin{array}{llll}\text { chemometric methods. } \quad \text { J. } & \text { Microchemical, } & \text { 58(3), }\end{array}$ http://dx.doi.org/10.1006/mchj.1997.1560

Elexová, E., Némethová, D. (2003). The effect of abiotic environmental variables in the Danube macrozoobenthic communities. Limnologica, 33(4), 340-354. http://dx.doi.org/10.1016/S0075-9511(03)80028-2

Elliot, J. M. (2006). Critical periods in the life cycle and the effects of a severe spate vary markedly between four species of elmid beetles in a small stream. Freshwater Biology, 51(8), 1527-1542. http://dx.doi.org/10.1111/j.1365-2427.2006.01587.x

Elliot, J. M. (2008). The ecology of riffle beetles (Coleoptera: Elmidae). Freshwater Reviews, 1, 189-203. http://dx.doi.org/10.1608/FRJ-1.2.4

Engel, S. R., \& Voshell J. R. Jr. (2002). Volunteer biological monitoring: Can it accurately assess the ecological condition of streams? American Entomologist, 48(3), 164-177. http://dx.doi.org/10.1093/ae/48.3.164

Flores, M. J. L., \& Zafaralla, M. T. (2012). Macroinvertebrate composition, diversity and richness in relation to the water quality status of Mananga River, Cebu, Philippines. Philippine Scinece Letter, 5(2), 103-113.

Gasim, M. B., Sahid, I., Toriman, E., Pereira, J. J, Mokhtar, M., \& Abdullah, M. P. (2009). Integrated Water Resources Management and Pollution Sources in Cameron Highlands, Pahang, Malaysia. American-Eurasian J. Agric. \& Environ. Sci., 5(6), 725-732.

Grandjean, F., Momon, J., \& Bramard, M. (2003). Biological water quality assessment of the white-clawed crayfish habitat based on macroinvertebrate communities: Usefulness for its conservation. Bull. Fr. Péche Piscic, 370-371, 115-125. http://dx.doi.org/10.1051/kmae:2003007

Griffith, M. B., Kaufmann, P. R., Herlihy, A. T., \& Hill, B. H. (2001). Analysis of macroinvertebrate assemblages in relation to environmental gradients in Rocky Mountain streams. Ecol. Appl., $11, \quad$ 489-505. http://dx.doi.org/10.1890/1051-0761(2001)011[0489:AOMAIR]2.0.CO;2

Gooderham, J., \& Tsyrlin, E. (2003). A guide to the freshwater macroinvertebrates of temperate Australia: The waterbug book. Second Edition. Australia: Impact Printing.

Hamed Yazdia,Nematollah Jaafarzadeh and Banafsheh Zahraie. (2014). Relationship between benthic macroinvertebrate bio-indices and physicochemical parameters of water: a tool for water resources managers. Journal of Environmental Health Science and Engineering. 10, 12(1), 30. http://dx.doi.org/10.1186/2052-336X-12-30

Harper, P. P. (1973). Life histories of Nemouridae and Leuctridae in Southern Ontario (Plecoptera). Hydrobiologia, 41, 309-356. http://dx.doi.org/10.1007/BF00016624 
Howitt, J. B. (2008). Environmental factors influencing the abundance of sandflies (Austrosimulium australense (Simuliidae)). Ecology Programme, University of Otago, New Zealand. http://akoaotearoa.ac.nz/sites/default/files/IBL\%20-\%20Example_2.pdf Accessed on 2 October 2014.

Hynes, H. B. N. (1970). The Ecology of Running Waters. Toronto Press: Toronto University

Ismail, B. S., Ngan, C. K., Cheah, U. B., \& Wan Abdullah, W. Y. (2004). Leaching potential of pesticides in a vegetable farm in the Cameron Highlands, Malaysia. Bull. Environ. Contam. Toxicol., 72, 836-843. http://dx.doi.org/10.1007/s00128-004-0320-5

Karanth, K. R. (1987). Groundwater Assessment Development and Management. McGraw-Hill Publishing, New Delhi.

Kato, M. G., Mitsuhashi, H., Kohmatsu, Y., Miyasaka, H., Nozaki, K., \& Nakanishi, M. (2005). A seasonal change in the distribution of a stream-dwelling stonefly nymph reflects oxygen supply and water flow. Ecol. Res. 20, 223-226. http://dx.doi.org/10.1007/s11284-004-0029-2

Krenkel, P. A., \& Novotny, V. (1980).Water Quality Management. Academic Press Inc., New York .

Kohut, M. E. (2007). A study of riffle beetle behavior in the Checkhall River Coleoptera: Elmidae. Texas: A \& M University.

Kopciuch, R. G., Berecka, B., Bartoszewicz, J., \& Buszewski B. (2004). Some considerations about bioindicators in environmental monitoring. Polish. Journal of Environmental Studies, $13,453-462$.

Kucuk, S. (2008). The effect of organic pollution on benthic macroinvertebrate fauna in Kirmir Creek in the Sakarya Basin. Adü Ziraat Fakültesi Dergisi, 5(1), 5-12.

Kumaran S., \& Ainuddin A. N. (2006). Forest, Water and Climate of Cameron Highlands. In Chan, N.W. 2006. Cameron Highlands: Issues and Challenges in Sustainable Development. Universiti Sains Malaysia.

Kusza, I. C. (2005). Comparing modified biological monitoring working party score system and several biological indices based on macroinvertebrates for water-quality assessment. Limonologica, 35, 169-176. http://dx.doi.org/10.1016/j.limno.2005.05.003

Lafront, M. (1984). Oligochaete communities as biological descriptors of pollution in the fine sediments of rivers. Hydrobiologica, 115, 127-129. http://dx.doi.org/10.1007/BF00027906

Latha C, Thanga VSG. (2010). Macroinvertebrate diversity of Veli and Ladinamkulam Lakes, South Kerala, India. J. Environ Biol., 547, 543-547.

Lobo, H., \& Alves, R. G. (2011). Influence of body weight and substrate granulometry on the reproduction of Limnodrilus hoffmeisteri (Oligochaeta: Naididae: Tubificinae). Zoologia, 28(5), 558-564. http://dx.doi.org/10.1590/S1984-46702011000500002 


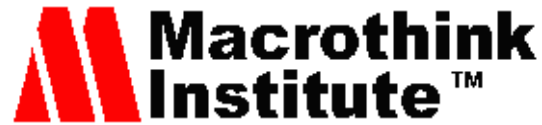

Loch, D. D., West, J. L., \& Perlmutter, D. G. (1996). The effect of trout farm effluent on the taxa richness of benthic macroinvertebrates. Aquaculture, 147, 37-55. http://dx.doi.org/10.1016/S0044-8486(96)01394-4

Martins, R. T., Stephan NNC., \& Alves, R. G. (2008). Tubificidae (Annelida: Oligochaeta) as an indicator of water quality in an urban stream in southeast Brazil. Acta Limnol. Bras., 20(3), 221-226.

Marques, M. M. G. S. M., Barbosa, F. A. R., \& Callisto, M. (1999). Distribution and abundance of Chironomidae (Diptera, Insecta) in an impacted watershed in South-East Brazil. Rev. Brasil Biol., 59(4), 553-561. http://dx.doi.org/10.1590/S0034-71081999000400004

Maul, J. D., Farris, J. L., Milam, C. D., Cooper, C. M., Testa, S., \& Feldam, D. L. (2004). The influence of stream habitat and water quality on macroinvertebrate communities in degraded $\begin{array}{lllll}\text { streams of northwest Mississippi. Hydrobiologia, } & \text { 518, }\end{array}$ http://dx.doi.org/10.1023/B:HYDR.0000025058.00605.6e

Miserendino, M. L., Brand, C., \& Prinzio, C. Y. D. (2008). Assessing urban impacts on water quality, benthic communities and fish in streams of the Andes Mountains, Patagonia $\begin{array}{lllll}\text { (Argentina). Water } \quad \text { Air } & \text { Soil } & \text { 91-110. }\end{array}$ http://dx.doi.org/10.1007/s11270-008-9701-4

Mohsen, Z. H., \& M. S. Mulla. (1982). The ecology of black flies (Diptera: Simuliidae) in some southern California streams. J. Med. Entomol., 19, 72-85. http://dx.doi.org/10.1093/jmedent/19.1.72

Moore, J. W. (1979). Influence of food availability and other factors on the composition, structure and density on a subartic population of benthic invertebrates. Hydrobiologia, 62, 215-223. http://dx.doi.org/10.1007/BF00043538

Muñoz, I., \& Prat, N. (1996). Effects of water abstraction and pollution on macroinvertebrate community in a Meditarranean River. Limnética, 12(1), 9-16.

Nemati Varnosfaderany M, Ebrahimi E, Mirghaffary N, Safyanian A. (2010). Biological assessment of the Zayandeh Rud River, Iran, using benthic macroinvertebrates. Limnol., 40, 226-232. http://dx.doi.org/10.1016/j.limno.2009.10.002

Ngan, C. K., Cheah, U. B., Wan Abdullah, W. Y., Lim, K. P., \& Ismail B. S. (2005). Fate of chlorothalonil, chlorpyrifos and profenofos in a vegetable farm in Cameron Highlands, Malaysia. Water, Air, and Soil Pollution Focus, 5, 125-136. http://dx.doi.org/10.1007/s11267-005-7408-8

Ogleni N, Topal B. (2011). Water quality assessment of the Mudurnu River, Turkey, using biotic indices. Water Resources Management, 25(11), 2487-2508. http://dx.doi.org/10.1007/s11269-011-9822-1

Pachón, R. T., \& Walton, W. E. (2011). Seasonal occurrence of black flies (Diptera: Simuliidae) in a desert stream receiving trout farm effluent. Journal of Vector Ecology, 36(1), 187-196. http://dx.doi.org/10.1111/j.1948-7134.2011.00156.x 
Paisley, M. F., Walley, W. J., Nikhade, J., \& Dils, R. (2003). Identification of the key biological indicators of nutrient enrichment in rivers for use in predictive/ diagnostics models. Diffuse Pollution Conference Dublin, (8A Ecology), 8-13.

Pliūraitè, V., \& Mickènienè, L. (2009). Benthic macroinvertebrate communities in agriculturally impaired streams. Environmental Research, Engineering and Management, 3(49), 10-20.

Ramirez, A., \& Pringle C, M. (1998). Invertebrate drift and benthic community dynamics in a lowland neotropical stream, Costa Rica. Hydrobiologia, 386, 19-26. http://dx.doi.org/10.1023/A:1003409927131

Revenga, C., Campbell, I., Abell, R., de Villiers, P., \& Bryer, M. (2005). Prospects for monitoring freshwater ecosystems towards the 2010 targets. Phil. Trans. R. Soc. B, 360, 397-413. http://dx.doi.org/10.1098/rstb.2004.1595

Sauter, G., \& Güde, H. (1996). Influence of grain size on the distribution of tubificid oligocahete species. Hydrobiologia 334, 97-101. http://dx.doi.org/10.1007/BF00017358

Singh K. P., Malik A., Mohan D., \& Sinha S. (2004). Multivariate Statistical Techniques for the Evaluation of Spatial and Temporal Variations in Water Quality of Gomti River (India): A Case Study. J. Water Research, 38(18), 3980-3992. http://dx.doi.org/10.1016/j.watres.2004.06.011

Swaminathan, M. S. M. (2003). Bio-diversity: An effective safety net against environmental pollution. Environmental Pollution. 287-291. http://dx.doi.org/10.1016/S0269-7491(03)00241-0

Tan, KW. (2010). Evaluation of social perception on water issues in Cameron Highlands (Malaysia) by Principal Factor Analysis. J. Environmental Science and Engineering, 4(4), 45-52.

Tan, K. W., \& Beh, W. C. (2015). Water Quality Monitoring Using Biological Indicators in Cameron Highlands Malaysia. Journal of Sustainable Development.Journal of Sustainable Development, 8(3), 28-42.

Tietze, N. S., and Mulla, M. S. (1989). Species composition and distribution of black flies (Diptera: Simuliidae) in the Santa Monica Mountains, California. Bull. Soc. Vector Ecol., 14, 253-261.

Wan Abdullah, W. Y., Aminuddin, B. Y., \& Zulkifli M. (2005). Modelling pesticide and nutrient transport in the Cameron Highlands, Malaysia agro-ecosystems. Water, Air, and Soil Pollution Focus, 5, 115-123. http://dx.doi.org/10.1007/s11267-005-7407-9

Yap, C. K., Rahim Ismail, A., Azrina, M. Z., Ismail, A., Tan, S. G. (2006). The influential of physico-chemical parameters on the distributions of Oligochateas (Limnodrilus sp.) at the polluted downstream of the Tropical Langat River, Peninsular Malaysia. J. Appl. Sci. Environ. Mgt., 10(3), 135-140. http://dx.doi.org/10.4314/jasem.v10i3.17332 
Yule, C. M., \& Yong H. S. (2004). Freshwater invertebrates of the Malaysian Region. Malaysia: Akademi Sains Malaysia.

Yong Cao, Anthony W. Bark \& W. Peter Williams. (1997). Analyzing benthic macroinvertebrate community changes along a pollution gradient: A framework for the development of biotic indices. Wat. Res., 31, 884-892. http://dx.doi.org/10.1016/S0043-1354(96)00322-3

Zwick, P. (2004). Key to the West Palaearctic genera of stoneflies (Plecoptera) in the larval stage. Limnologica, 34, 315-348. http://dx.doi.org/10.1016/S0075-9511(04)80004-5

\section{Copyright Disclaimer}

Copyright for this article is retained by the author(s), with first publication rights granted to the journal.

This is an open-access article distributed under the terms and conditions of the Creative Commons Attribution license (http://creativecommons.org/licenses/by/3.0/). 